INPLASY

PROTOCOL

To cite: Yao et al. Targeted Temperature Management for In-Hospital Cardiac Arrest: a meta-analysis. Inplasy protocol 2021110021. doi:

10.37766/inplasy2021.11.0021

Received: 06 November 2021

Published: 06 November 2021

Corresponding author:

Hui-Bin Huang

hhba02922@btch.edu.cn

Author Affiliation:

Beijing Tsinghua Chang Gung

Hospital, Tsinghua University.

Support: None.

Review Stage at time of this submission: Data analysis.

Conflicts of interest:

None declared.

\section{Targeted Temperature Management for In-Hospital Cardiac Arrest: a meta-analysis}

Yao, Y1; Duan, JY2; Qin, JP3; Huang, HB4.

Review question / Objective: To investigate the effectiveness and safety of targeted temperature management (TTM) in spatients after in-hospital CA (IHCA).

Eligibility criteria: We included published studies if they met the following criteria: (1) RCT or any two-group comparison studies; (2) Studies focused on IHCA survivors, without restrictions of initial rhythm, the timing of TTM or cooling methods; (3) Included patients were supported with or without TTM; (4) Studies should report the outcome of survival.

INPLASY registration number: This protocol was registered with the International Platform of Registered Systematic Review and Meta-Analysis Protocols (INPLASY) on 06 November 2021 and was last updated on 06 November 2021 (registration number INPLASY2021110021).

\section{INTRODUCTION}

Review question / Objective: To investigate the effectiveness and safety of targeted temperature management (TTM) in spatients after in-hospital CA (IHCA).

Condition being studied: In-Hospital Cardiac Arrest. Our team members come from a tertiary hospital in China and all the members have extensive experience in patients with CA. Moreover, our team members have published several metaanalyses, which can guarantee the completion of the current study.

\section{METHODS}

Participant or population: In-hospita cardiac arrest (IHCA) adult patients. 
Intervention: Managed with targeted temperature management

Comparator: CA patients managed without targeted temperature management.

Study designs to be included: Studies were included if they reported data on predefined outcomes in adult IHCA survivors managed with TTM.

Eligibility criteria: We included published studies if they met the following criteria: (1) RCT or any two-group comparison studies; (2) Studies focused on IHCA survivors, without restrictions of initial rhythm, the timing of TTM or cooling methods; (3) Included patients were supported with or without TTM; (4) Studies should report the outcome of survival.

Information sources: PubMed, Cochrane Controlled Trials Register, and Embase databases.

Main outcome(s): The primary outcome and secondary outcome was mortality rate and good neurological outcome at the longest followed-up available, respectively.

Quality assessment / Risk of bias analysis: Included studies were appraised for their risk of bias using the Cochrane Collaboration tool to assess the risk of bias in RCTs and the Newcastle-Ottawa scale for assessing the risk of bias in observational studies.

Strategy of data synthesis: To obtain more robust results, we used random-effects models as pooling method for all the measuring.

Subgroup analysis: We further conducted subgroup analyses to test the robustness of the outcomes basing on the important clinical features (i.e., country, sample size, design, and study start time).

Sensitivity analysis: Sensitivity analyses were performed by excluding trials that potentially biased the results.
Keywords: in-hospital cardiac arrest; cardiac arrest; targeted temperature management; neurological outcome; metaanalysis.

Contributions of each author:

Author 1 - Yan Yao.

Author 2 - Jing-Yi Duan.

Author 3 - Jun-Ping Qin.

Author 4 - Hui-Bin Huang.

Country(ies) involved: China. 